\title{
Development of an One-item Question to Assess Spiritual Well-being in Advanced Cancer Inpatients in South Korea
}

\author{
Sang-Yeon Suhab, Youngmin- Parkc, Sun Hyun Kim ${ }^{\text {d }}$, Eon Sook Lee ${ }^{e}$, Jeanno Parkf, Seok Joon Yoong, Yu Jung Kimh, Beodeul \\ Kang', Jung Hye Kwon', Kwonoh Park', Hong-Yup Ahn', David Hui'
}

${ }^{a}$ Department of Family Medicine, Palliative Care Center, Dongguk University Ilsan Hospital, Goyang, South Korea; ${ }^{b}$ Department of Medicine, School of Medicine, Dongguk University, Seoul, South Korea; cDepartment of Family Medicine, National Health Insurance Service Ilsan Hospital, Goyang, South Korea; ${ }^{d}$ Department of Family Medicine, Catholic Kwandong University International St. Mary's Hospital, Incheon, South Korea; ${ }^{\mathrm{e} D e p a r t m e n t ~ o f ~ F a m i l y ~ M e d i c i n e, ~ P a l l i a t i v e ~ C a r e ~ C e n t e r, ~ I n j e ~}$ University Ilsan Paik Hospital, Goyang, South Korea; fDepartment of Internal Medicine, Bobath Hospital, Seongnam, South Korea; sDepartment of Family Medicine,

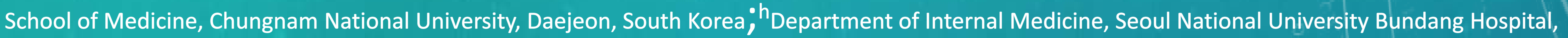
Seongnam, South Korea;iDivision of Medical Oncology, Department of Internal Medicine, Yonsei University College of Medicine, Seoul,South Korea;jDepartment of Internal Medicine, Kangdong Sacred Heart Hospital, Hallym University, Seoul, South Korea; ${ }^{k}$ Department of Internal Medicine, Pusan National University Yangsan Hospital, Pusan, South Korea; 'Department of Statistics, Dongguk University, Seoul, South Korea; ${ }^{\mathrm{m}}$ Department of Palliative Care and Rehabilitation Medicine, Division of Cancer Medicine, The University of Texas MD Anderson Cancer Center, Houston, TX, United States

Background Spiritual well-being (SWB) is important for patients having life-limiting illness. Functional Assessment of Chronic Illness Therapy-Spirituality 12(FACIT-Sp 12) is commonly used to assess spiritual well-being(SWB). Shortened versions of question would be helpful to assess SWB in emaciated patients. Our goal was to develop an one-item question appropriately in order to assess SWB in advanced cancer inpatients.

Methods

This is a cross-sectional, multicenter study involving adult advanced cancer inpatients from 7 palliative care units in South Korea. Candidates of an one-item question were three questions to check; their peacefulness(Are you at peace?), spirituality(Do you think yourself as a spiritual person?) and religiosity(Do you think yourself as a religious person?). Patients answered three questions by self-rating using numeric rating scales(0-10). We collected data on clinicodemographic characteristics and SWB. SWB was measured by FACIT-Sp 12, comprised of 2 subscales (Meaning/Peace and Faith). Pearson's correlation test was conducted to determine the relationship among three questions, the total score of FACIT-Sp 12 and its subscales.

Results A total number of 194 patients were enrolled in the study. A good correlation was observed in the questions of spirituality $(r=0.732, P<0.01)$ and religiosity $(r=0.708, P<0.01)$ with a total score of FACIT-Sp 12. Question of peacefulness showed a moderate correlation with a total score of FACIT-Sp 12( $r=0.505, \mathrm{P}<0.01)$. The faith subscale showed a good correlation with the questions of spirituality $(r=0.790, P<0.01)$ and religiosity $(r=0.758, P<0.01)$. All three questions had a moderate correlation with the meaning/peace subscale.

Conclusion The questions of spirituality and religiosity showed better convergence validity than the question of peacefulness. Therefore, we recommend the questions of spirituality or religiosity as an one-item question to assess SWB in advanced cancer inpatients.

Table 1. Correlations of One Item Questions between FACIT-Sp12 $(n=194)$

\begin{tabular}{|c|l|c|c|}
\hline One Item Questions & \multicolumn{1}{|c|}{ FACIT-Sp12 } & $\begin{array}{c}\text { Pearson's correlation } \\
\text { coefficient }\end{array}$ & P-value \\
\hline \multirow{2}{*}{ Peacefulness Q } & Total Score & 0.505 & $<0.001$ \\
& Meaning/Peace & 0.577 & $<0.001$ \\
\cline { 2 - 3 } & Faith & 0.334 & $<0.001$ \\
& Total Score & 0.732 & $<0.001$ \\
\hline \multirow{2}{*}{ Spirituality Q } & Meaning/Peace & 0.475 & $<0.001$ \\
& Faith & 0.790 & $<0.001$ \\
& Total Score & 0.708 & $<0.001$ \\
\hline \multirow{2}{*}{ Religiosity Q } & Meaning/Peace & 0.466 & $<0.001$ \\
& Faith & 0.758 & $<0.001$ \\
\hline
\end{tabular}

P-values were obtained by Pearson's correlation.

FACIT-Sp 12: Functional assessment of chronic illness therapy-spirituality 12; Total score: Spiritual wellbeing was measured by total sum of FACIT-Sp 12; Meaning/peace: sum of Q1 through Q8; Faith: sum of Q9 through Q12.

Peacefulness Q: Are you at peace?; Sprituality Q: Do you consider yourself as a spiritual person?; Religiosity Q: Do you consider yourself as a religious person?
Figure 1. Diagram of Relationship between One Item Questions and FACIT-Sp12

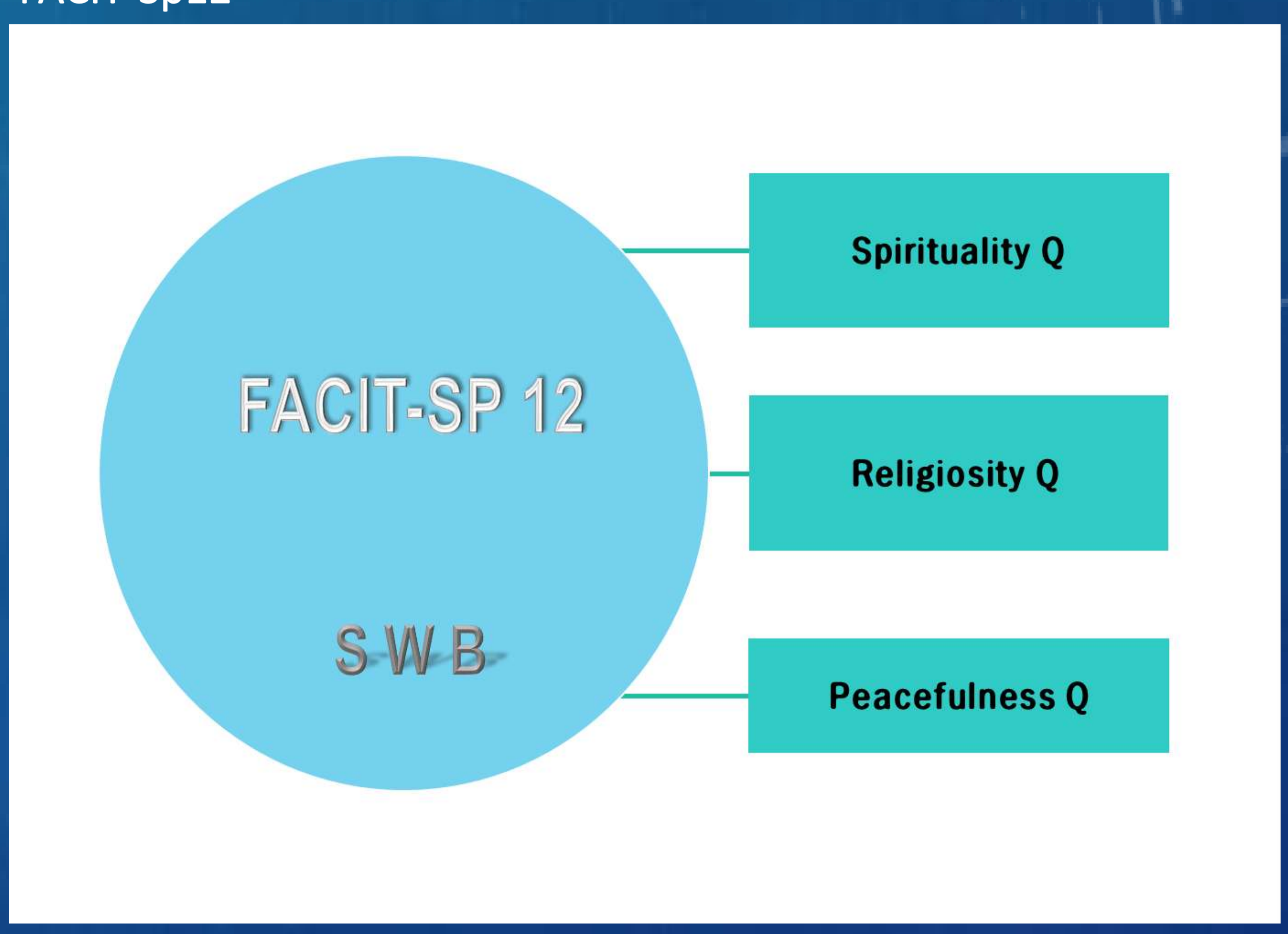

FACIT-Sp 12: Functional assessment of chronic illness therapy-spirituality 12 SWB: Spiritual well-being

Sprituality Q: Do you consider yourself as a spiritual person?; Religiosity Q: Do you consider yourself as a religious person?; Peacefulness Q: Are you at peace? 